Session 12-11

\title{
Simulation of the Electric Field and Potential for a Micro Pattern X-ray Detector Using Maxwell Software
}

\author{
Jamel Gray, Derrek Anderson, and E. H. Shaban \\ Southern University \\ Electrical Engineering Department \\ Baton Rouge, LA 70813 \\ D. P. Siddons and A. Kucsweski \\ Brookhaven National Lab \\ Upton, NY 11973
}

\begin{abstract}
In the summer of 2007, the faculty and student team (FaST) program from Southern University in Baton Rouge, Louisiana supported by NSF, DOE, and LS-LAMP conducted a detailed study to design, simulate, build and test a micro-pattern x-ray fluorescence gas detector at Brookhaven National Laboratory (BNL). We used AutoCAD to design the detector's parts that were machined and assembled to form the proposed detector. We have used Maxwell software to predict the electrical field and potential in the drift and amplification regions of the detector. This paper describes the hands on learning process and in depth research accomplishment that the undergraduate students have undertaken in the ten weeks of intensive summer learning. A detailed Maxwell simulation for the gas electron multiplier (GEM) and the micro mesh gaseous (MICROMEGAS) detectors are shown and experimental results of the double GEM fluorescence $\mathrm{x}$-ray detector are depicted.
\end{abstract}

\section{Introduction}

The Faculty and Student Team (FaST) consisted of Dr. E. H. Shaban, Electrical Engineering Department, Southern University Baton Rouge, and two students: Mr. Derrek Anderson and Mr. Jamel Gray. The FaST program from Southern University has worked at Brookhaven National Lab (BNL) with the same mentor, Dr. D. P. Siddons at the National Synchrotron Light Source (NSLS) for the previous three summers. Each summer, the X-Ray detector has been developed, improved, and tested. There was a remarkable improvement each summer that made the detector more competitive as compared with the traditional fluorescence's detectors.

We have designed the detector using AutoCAD and used the machine shop at NSLS to build the detector using plexi glass, PCB, and Mylar, leak proof gas inlets, and gaskets. The student version of the Ansoft corporation program Maxwell was used to predict and to plot the distribution of the electric filed and the potential for the gas electron multiplier (GEM). The GEM is made up of fifty microns thick Kapton insulator that is covered by five microns thick copper in both sides. Holes of 70-90 microns diameters and 120-140 microns pitch are

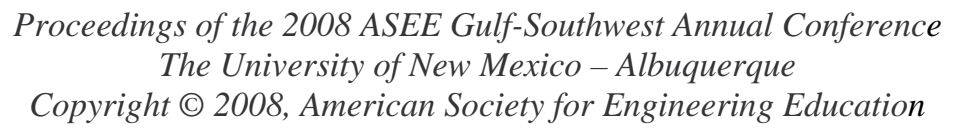


chemically etched in both sides of the copper through the insulator. The function of the GEM is to create strong electric field that allows the electrons ionized by x-ray in the drift region to accelerate and multiply by impact ionization. This amplification within the GEM is the key factor for providing amplification of charge without an introduction of electronic noise. This has produced enhanced amplification of the ionized electrons during extended x-ray absorption fine structure (EXAFS). We also used the AutoDesk Inventor to provide a three dimensional model of the designed x-ray fluorescence detector. Last summer we have replaced the GEM by a micro mesh gas detector (MICROMEGAS). The MICROMEGAS consists of steel mesh that is separated from a PCB by 100-150 microns of Kapton or insulator pillars. The region between the metallic mesh and the PCB has a very high electric filed that allows electron multiplication using impact ionization. We are predicting that the MICROMEGAS can provide better amplification and better signal to noise ratio than the GEM fluorescence's detector.

\section{AutoCAD}

We used the AutoCAD program to provide different views of the GEM and MICROMEGAS xray detectors. A two dimensional plan views of the pieces of the detector that form the Myler window cover, the drift region with inlet and outlet of the gas, the amplification region where the GEM is attached and fixed, and the collection region where the total charge is collected. The visuals can be seen in figures 1 and figure 2. In this detector we used a double GEM detector to enhance the amplification and to decrease the probability of sparks. The visuals of the different regions have very discrete values of all of the dimensions to our detectors. From these pictures and dimensions for our project, we were able to build our actual parts in the NSLS User Shop. The parts that were used were assembled out of various different materials as shown in figure 3. After building the parts, the X-Ray detectors were assembled together and were ready for the testing phase.

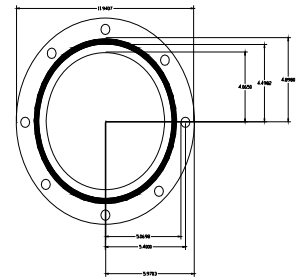

Figure 1. Largest housing with gas inlet and gas outlet

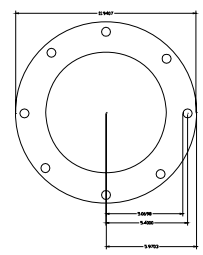

Figure 2. Flange with a GEM on top with PCB Anode at bottom

Proceedings of the 2008 ASEE Gulf-Southwest Annual Conference

The University of New Mexico - Albuquerque

Copyright (C) 2008, American Society for Engineering Education 


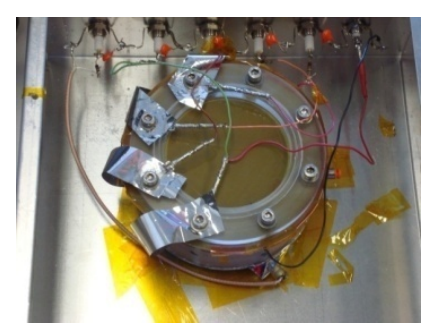

Figure 3. Assembled GEM detector

\begin{abstract}
Autodesk Inventor
Autodesk Inventor software is the foundation for digital prototyping. The Inventor model created is an accurate 3D digital prototype that enables users to validate design and engineering data as they work, to minimize the need for physical prototypes, and reduce costly engineering changes discovered after the design is sent to be manufactured. Innovative approaches are used to accelerate and simplify the concept-to-manufacturing process. We used Autodesk inventor to illustrate the design of the Gas Electron Multiplier (GEM) X-Ray Fluorescence Detector, and the MICROMEGAS Detector. A typical build up of MICROMEGAS is shown in figure 4.
\end{abstract}

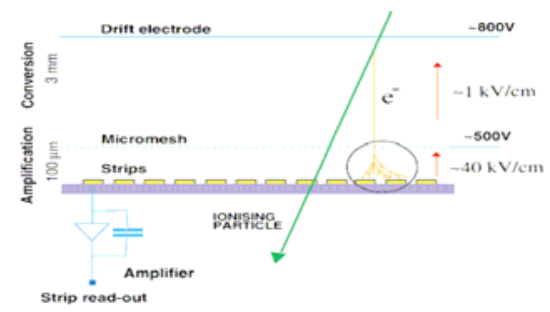

Figure 4. Schematic of MICROMEGAS

Figures 5 displays the Printed Circuit Board (PCB) and rear view of the X-Ray Fluorescence detector for the full x-ray fluorescence detector. Figure 6 shows the Mylar and front view of the detector. The Mylar window allows the x-ray to penetrate the detector.

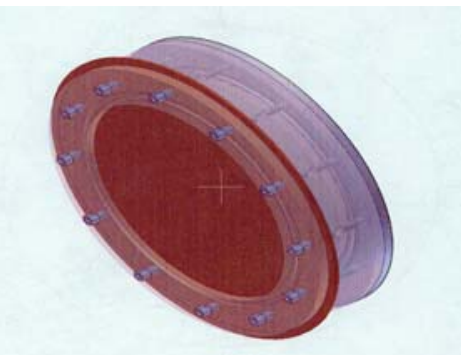

Figure 5. GEM Assembly using Inventor-PCB side 


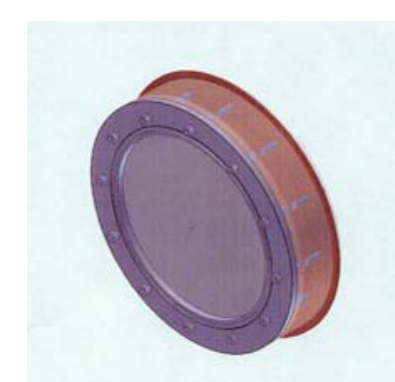

Figure 6. GEM Assembly using Inventor-Mylar Side

\section{Maxwell}

We have used the Maxwell program to plot both the electric field and the potential for the X-ray detector using the gas electron multiplier (GEM) and the MICROMEGAS. Maxwell software solves complicated equations and plots the results showing the electric field and the potential for various configurations. Fig $7 \& 8$ show the results obtained when a voltage difference is applied between the metallic mesh of the MICROMEGAS and the PCB. This is the region where the main charge amplification takes place. There is another drift field which is applied between the Mylar and the metallic mesh. The potential distribution is also depicted for MICROMEGAS.

Similar results were obtained for the GEM detector in Figs 9 \&10. We have used the Maxwell program to plot both the electric field and the potential for the X-ray detector using the gas electron multiplier (GEM). Voltages are applied at the Mylar, the top of the GEM and the bottom of the GEM to vary both the drift region electric field where the X-ray ionizes the gas. A voltage difference is applied across the GEM to increase the electric field in order to accelerate the velocity of the electrons to produce the avalanche multiplication. There is no voltage applied in the PCB where the electrons are collected.

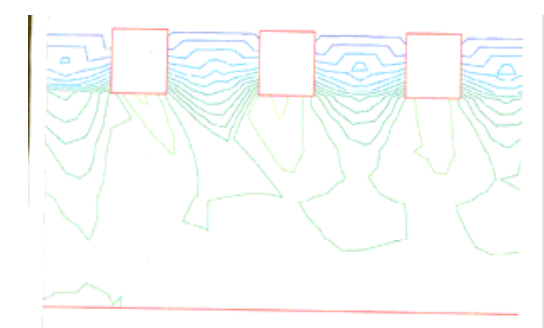

Figure 7. Maxwell Simulation for the Electric Field for MICROMEGAS

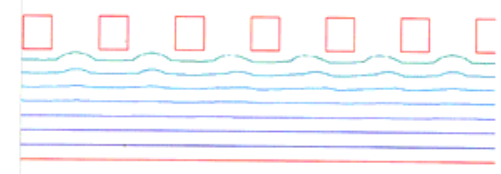

Figure 8. Maxwell Simulation for Potential in MICROMEGAS

Proceedings of the 2008 ASEE Gulf-Southwest Annual Conference

The University of New Mexico - Albuquerque

Copyright (C) 2008, American Society for Engineering Education 


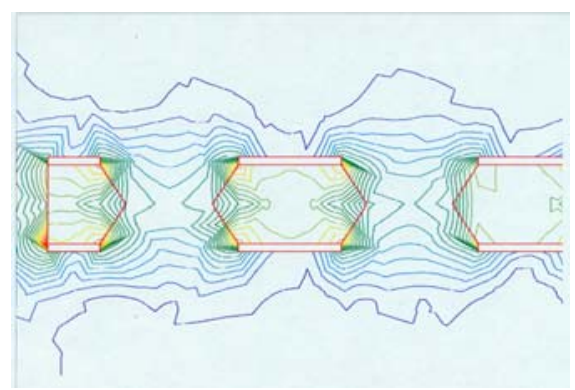

Figure 9. Maxwell Simulation of Electric Field Across GEM

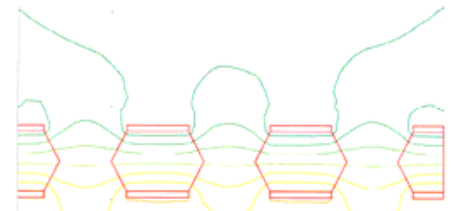

Figure 10. Maxwell Simulation of the Potential across GEM

\section{Results}

Our Single GEM and Double GEM Fluorescence X-Ray Detectors were connected to Argon Carbon Dioxide gas with (80\%/20\%) mixture at X-ray beamline. We used DC high voltage power supply to provide voltages to the Mylar, the top of the GEM and the bottom of the GEM. The main function of the DC high voltages is to provide a constant Electric field. The anode or the PCB was at virtual ground and is directly connected to the Keithley 428 amplifier. The output of the charge amplifier is connected to a voltage to frequency converter. The output from the converter was then interpreted in counts using computer software. This allows the display of the absorption edge as a function of the x-ray energy. The detector that was tested first was the Single GEM detector. The starting voltages applied to the detector were 2050V on the Mylar, $600 \mathrm{~V}$ on the TGEM, and 350V on the BGEM. The voltage potential between the Mylar and the TGEM was kept constant at $1450 \mathrm{~V}$ all the time during the measurement to keep the drift field constant. This test was run for 11 scans while increasing the voltages on the Mylar and TGEM by $25 \mathrm{~V}$ each time. The potential between the TGEM and BGEM was changed continuously to increase the electric field for the maximum gain per scan.

The fluorescence scans for a manganese sample using a single GEM are shown in Fig. 11 and a similar scans for manganese using a double GEM are shown in Fig. 12. For the Single GEM, the gain as a function of the applied voltage is shown in Fig. 13 and a similar plot for the gain of a double GEM is shown in Fig. 14. The comparison of the fluorescence scan for manganese using the single and the double GEM were depicted in Fig. 15. This shows the remarkable amplification obtained using the double GEM as compared to the single GEM. 
A typical scan using a double GEM for an arbitrary tree leaf indicates the detection of a manganese and iron within the leaf of the tree as in Fig. 16 and 17. This is the first time we were able to detect manganese using the double GEM detector. We did not succeed in detecting manganese using the single GEM detector. Although we were able to build and test and the MICROMEGAS detector, we were able to make one or two scans after which the MICROMEGAS experienced a short. We intend to rebuilt the MICRPMEGAS detector and repeat the measurements in the near future. The student's team who accomplished building the detector are shown in Fig. 18.

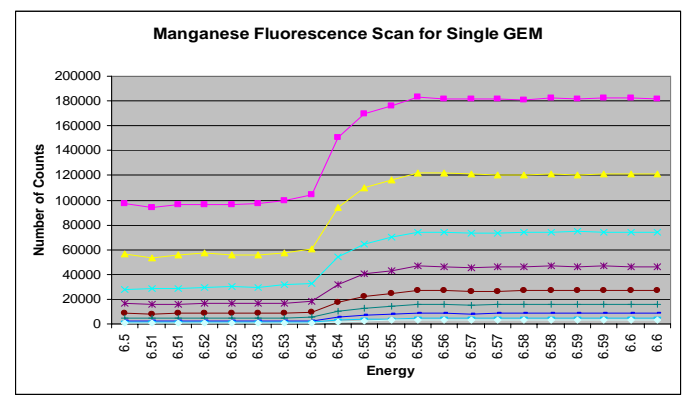

Figure 11. Fluorescence Scan for Manganese Using Single GEM Detector

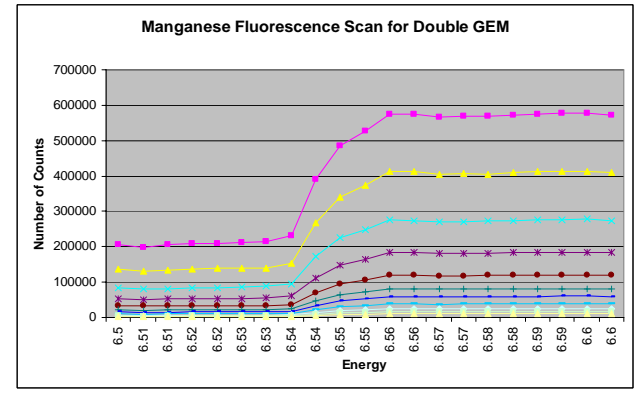

Figure 12. Fluorescence Scan for Manganese Using Double GEM

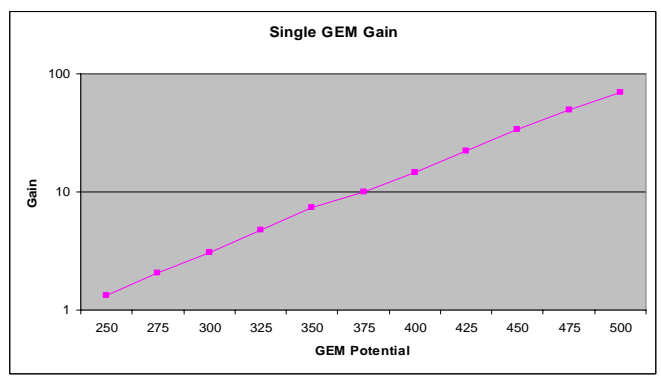

Figure 13. Gain of Single GEM 


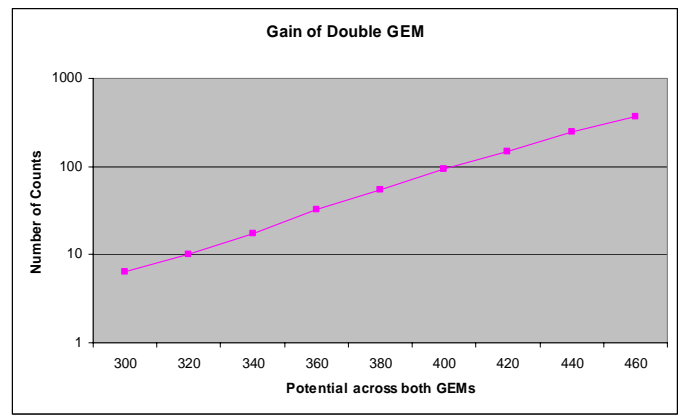

Figure 14. Gain of Double GEM

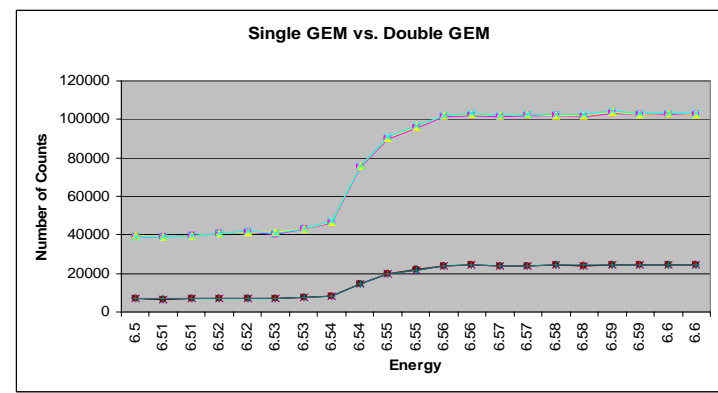

Figure 15. Single GEM Detector vs. Double GEM detector

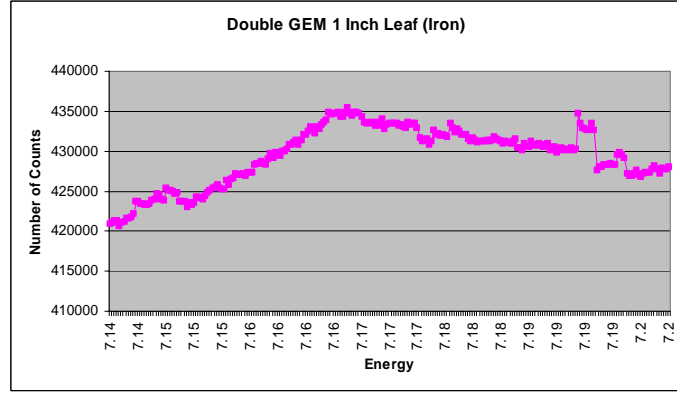

Figure 16. Double GEM Iron

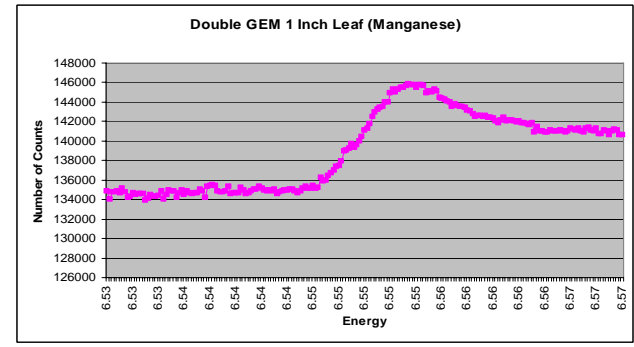

Figure 18. Double GEM Manganese

\section{Summary and Conclusions}

The two X-Ray detectors single GEM and double GEM, were designed, machined and tested using fluorescence $\mathrm{X}$-ray for Mn samples with an absorption edge of $6.539 \mathrm{KeV}$. The Single

Proceedings of the 2008 ASEE Gulf-Southwest Annual Conference

The University of New Mexico - Albuquerque

Copyright (C) 2008, American Society for Engineering Education 
GEM detector provided a good gain of 90 with a voltage across the GEM of $500 \mathrm{~V}$. Also the Double GEM detector provided a good gain of 400 with a voltage across both GEMs of $460 \mathrm{~V}$. After both detectors were tested, it was found that the Double GEM detector had about four times a greater gain than the Single GEM, which entails that the Double GEM detector is a better detector to use to receive a higher gain. We were able detect dilute amounts of $\mathrm{Mn}$ and Fe from a tree leaf using our Double GEM detector.

\section{Acknowledgments}

We would like to thank NSF, the director and personnel of LS-LAMP at Southern University Baton Rouge, DOE, Office of Science Education, and NSLS at Brookhaven National Lab, for funding and providing the opportunity to conduct high quality research during the summer of 2007. We would like to thank 3M Corporation for supplying the GEM.

\section{References}

[1] Sauli, F. "GEM: A new concept for electron amplification in gas detectors." Nuclear Instruments \& Methods in Physics Research (1997) 531-534.

[2] DiJulio, D. D., A.I. Hawari, R. Berliner. "A Gd-based gaseous electron multiplier detector for neutron scattering applications." Nuclear Instruments \& Methods in Physics Research (2007) 1-4.

$<$ www.sciencedirect.com>.

[3] Eric Huey, Marcus Mason, “Development of Single and Double GEM (Gas Electron Multiplier) Based Detectors,” (to be published in Journal of Undergraduate Research)

[4] R. Bouclier, M. Capeans, W. Dominick, M. Hoch, J-C. Labbe’, G. Million, L. Ropelewski, G. Sauli, and A. Sharma, "The Gas Electron Multiplier (GEM),"

CERN CH-112 Geneva, Switzerland

\section{DERREK ANDERSON}

Derrek Anderson is a senior student in the Electrical Engineering Department at Southern University in Baton Rouge, LA. He is planning to graduate this upcoming summer but is exactly unsure of what his next plans are. The top of his choice is to pursue his graduate degree, starting next spring.

\section{JAMEL GRAY}

Jamel Gray is a senior Electrical Engineering student at Southern University and A \& M College in Baton Rouge, $\mathrm{La}$. After receiving his undergraduate degree he is planning to venture out into the corporate world, but he plans to pursue a graduate degree in Electrical Communication Systems shortly after working.

\section{E. H. SHABAN}

Dr. E. H. Shaban is a graduate of the University of Florida, Gainesville in 1986. He has worked as a visiting professor at the University of Puerto Rico, Mayaguez from1987-88 academic year. He worked briefly in a small family oriented company in Gainesville, Fl. After which he joined the Electrical Engineering department at Southern University Baton Rouge in January of 1991. Dr. Shaban is an associate professor in Electrical Engineering.

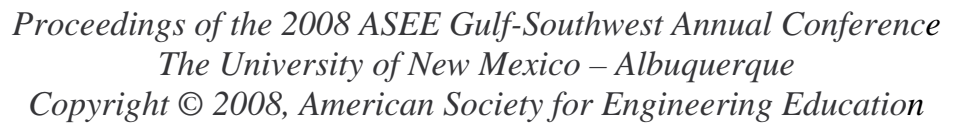


D. P.. SIDDONS

Dr. Siddons is the head of detectors and control group for the beamline at NSLS, BNL.

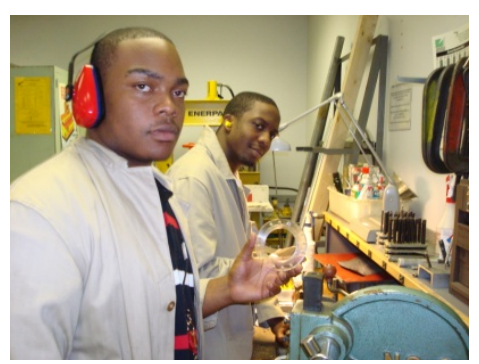

Figure 18. Student Team Assembling Detector

Proceedings of the 2008 ASEE Gulf-Southwest Annual Conference The University of New Mexico - Albuquerque

Copyright $\left({ }_{0}\right.$ 2008, American Society for Engineering Education 